\title{
Integrated primary care-behavioral health program development and implementation in a rural context
}

\author{
Kendra Campbell ${ }^{1}$, Loren McKnight ${ }^{1}$, Angel R. Vasquez ${ }^{1}$
}

\section{Abstract}

Objective: Despite the known benefits of integrated primary care and behavioral health services, integrated behavioral health services have not been readily used in medical clinics in interior Alaska. With minimal resources, we recently developed an integrated primary care-behavioral health program in a medical clinic in interior Alaska to meet clinic and community needs. The objective of this study was to explore initial program outcomes and determine the feasibility of program development and implementation.

Methods: We initially conducted a needs assessment for integrating behavioral health services into primary care. Program development was informed by specific clinic needs. Following program implementation, initial program outcomes were tracked with use of data from the electronic health record and patient and provider use and satisfaction surveys. The level of integration of primary care and behavioral health services was evaluated with the Practice Integration Profile.

Results: A total of 188 patients were seen by behavioral health consultants during the initial pilot phase, including $44.0 \%$ referred for mental health symptoms, $33.1 \%$ referred for physical health issues, and $22.3 \%$ referred for both mental and physical health issues. The initial program outcomes indicate modest clinical improvement (measured by the nine-item Patient Health Questionnaire) as well as patient and provider satisfaction with the model, and a moderate level of practice integration.

Conclusion: On the basis of the initial findings, it appears that our integrated primary carebehavioral health program has the potential to serve an important role in addressing the behavioral health needs of the local population. Our implementation procedure and initial program outcomes suggest that such models are feasible in rural and small-scale settings with minimal overhead costs.

Keywords: Integrated healthcare; primary care; behavioral health; program development; rural healthcare

Significance statement: Integrating behavioral health services into primary care medical settings offers a variety of benefits, including improved continuity of care and access to service, more effective health prevention and management, and clinical cost-effectiveness. Integrated models have not been readily used in rural US settings. With minimal resources, we developed an integrated primary care-behavioral health program in the family medicine department of a semirural medical clinic in Alaska to meet clinic and community needs. The initial program outcomes suggest that this integrated primary care-behavioral health program is helping to address the behavioral health needs of the local population. Our findings suggest that integrated healthcare models are feasible in rural or small-scale settings.
1. Department of Psychology, University of Alaska Fairbanks, Fairbanks, AK, USA

CORRESPONDING AUTHOR:

Kendra Campbell, $\mathrm{PhD}$ Department of Psychology, University of Alaska Fairbanks, PO Box 756480, Fairbanks, AK 99775-6480, USA

E-mail: kendra.campbell@alaska.edu

Received 24 February 2018; Accepted 28 June 2018 


\section{Introduction}

As is becoming readily apparent in the healthcare literature, integrating behavioral health services into primary care medical settings offers multiple benefits in regard to clinical cost-effectiveness, continuity of patient care, and more effective prevention and management of a wide array of physical and mental health concerns [1-4]. Integration of behavioral health services within primary care is designed to address the behavioral health needs of patients who otherwise may not be seen in a specialty mental health clinic. Integrated primary care and behavioral health services can reduce barriers to accessing mental health services, improve patient health outcomes, and facilitate interprofessional dialogue $[1,5]$. Primary care is an opportune setting to screen patients for and facilitate treatment of behavioral health issues that may be impacting patient health outcomes, and inclusion of integrated behavioral health services is becoming a crucial factor in offering a more holistic patient treatment [6].

Despite the known benefits, fully integrated behavioral health services have not been readily used in rural medical clinics in the United States. The interior region of Alaska is composed of rural villages and small communities, with the largest population living in the region's largest town, which is relatively small and very remote. Almost all the residents of the interior living rurally must travel to town for medical services and behavioral health treatment. However, there is limited access to health services. It is important to maximize face-to-face interactions and provision of services to patients seeking treatment in this community.

We recently developed an integrated primary care-behavioral health program in the family medicine department of a medical clinic in interior Alaska to meet clinic and community needs. Included in the current report is our outlined process from development to implementation along with initial outcomes from the 9-month pilot phase. The initial goals for program development and implementation were to collaborate with local healthcare organizations to conduct needs assessments for integrating behavioral health services into primary care. Ongoing program development and evaluation include tracking patient use of, access to, and satisfaction with behavioral health services, tracking patient clinical outcomes and identifying specific outcomes that should continue to be monitored as part of ongoing evaluation, and assessing provider satisfaction with the integrated primary care-behavioral health model. Future goals include continued evaluation of the integrated primary care-behavioral health program, including specific health outcomes and treatment cost-effectiveness. Our goal is to effect positive change in physical and behavioral healthcare delivery in this region and to provide more effective service management in rural healthcare facilities.

\section{Methods}

\section{Program development}

Setting: Our program development occurred within one of the main private outpatient medical clinics in the community with a borough population of approximately 100,000 people. The family medicine department includes 14 primary care providers (physicians and physician assistants) and serves approximately 13,000 patients. The research methods used were approved by the Institutional Review Board at the University of Alaska Fairbanks.

Needs assessment: The first step of our process in developing a novel integrated primary care-behavioral health program in interior Alaska was to conduct a needs assessment to determine the particular needs and perspectives within one of the main outpatient medical clinics in the community. We surveyed primary care staff to assess their perceived need for and use of integrated behavioral health providers in the clinic. According to our assessment, primary care providers estimated that on a typical day up to $50.0 \%$ of their scheduled patients present with a mental health concern; for approximately $30.0 \%-50.0 \%$ of these patients, the mental health issue was the sole or primary concern, and for approximately $90.0 \%-100.0 \%$ of these patients, the mental health issues were exacerbating medical issues and/or impacting their medical treatment. Further, providers believed that almost all patients could benefit from meeting with a behavioral health provider for either a mental health issue or a medical issue for which a behavioral intervention would be indicated. Our initial assessment findings suggested a definite need for integrated behavioral health services in primary care clinics in interior Alaska, especially since access to mental health services is limited in such a remote community. Encouragingly, from these results, local 
medical providers appeared to be very motivated to include integrated behavioral health services in their practice.

Current model: After gaining buy-in from the clinic providers and administration, we sought to develop a level 4 colocated primary care behavioral health model [7] to meet the clinic needs. In this model, the physical workspace and patient care systems (e.g., electronic health record [EHR]) are integrated and provider communication and collaboration is face-to-face. This model was favorable to the clinic because it maximized existing resources (e.g., workspace) without implementing a more widespread change to the clinic practice and flow (e.g., by adding morning huddles or integrated case conferences). Notably, the primary care providers' recognized need for integrated behavioral health services (as discussed earlier) was helpful in obtaining organizational support.

To distinguish our integrated program from the concurrently implemented specialty behavioral health service in the clinic (staffed by two behavioral health clinicians), we labeled our program Family Medicine-Behavioral Health (FMBH) and our clinicians are described as behavioral health consultants (BHCs). The model was developed and implemented with minimal resources needed from the clinic itself. The initial phase, a 9-month pilot, consisted of one lead psychologist with specialty training in clinical health and primary care psychology who was contracted to train two to four doctoral-level psychology trainees. Because the pilot FMBH program includes trainees, patients are not billed for services.

The FMBH program is a consultant model in which BHCs are colocated in the primary care clinic and available for sameday, warm handoff referrals from primary care providers. The behavioral health interventions in this setting are designed to augment usual primary care health treatment and prevention. Behavioral health visits in this model are brief and specific to the referral concern. Clinical service delivery in our FMBH model includes traditional mental health services (e.g., brief, evidence-based intervention and assessment for depression, anxiety, substance use), crisis management prevention and intervention (e.g., suicide risk assessments), evidence-based behavioral medicine interventions (e.g., behavioral pain management, chronic disease management, weight management, stress management, smoking cessation interventions), and collaboration/consultation with medical providers regarding patient care (e.g., facilitation of longer-term mental health referral; psychotropic medication risk management). All behavioral health services are delivered in the primary care setting (i.e., in one of the clinic's examination rooms), and referrals are primarily via warm handoff from the primary care provider in the context of a normal primary care visit. In our initial phase, we staffed the clinic with BHCs for only 3-4 days a week, and so also accepted electronic referrals during our pilot.

\section{Initial outcomes measures}

EHR tracking system: To track and monitor how the pilot FMBH program is working, we developed a database using the EHR to track clinic- and patient-specific outcomes. This outcome tracking included reasons for referral and patients' before and after scores on the nine-item Patient Health Questionnaire (PHQ-9) [8]. We began administering the PHQ-9 to all referred patients partway through the initial program implementation. See Appendix A for a full breakdown of the data tracked through the EHR.

Patient feedback: Feedback from patients was requested after the initial warm handoff encounter about their experience on a short paper survey dropped off anonymously at the reception desk. Most items were on a five-point Likert scale and included questions such as "Did your primary care provider and behavioral health consultant work well together?" and "Did the behavioral health consultant understand your problem?" See Appendix B for the full survey.

Provider feedback: Provider surveys were collected by self-report surveys via https://www.qualtrics.com/. Initial provider feedback included use of and satisfaction with the model. Most items were on a five-point Likert scale and included questions such as "How satisfied are you with the accessibility of the Family Medicine-Behavioral Health team?" and "How satisfied are you with the care provided for your patients by the Family Medicine-Behavioral Health team?" See Appendix C for the full survey.

Practice integration: To determine the current level of integration between primary care and behavioral health 
services in our FMBH model, the Practice Integration Profile (PIP) [9] was administered to the lead behavioral health clinician and clinic medical director. The PIP is a self-assessment of practice integration based on the Agency for Healthcare Research and Quality's Lexicon for Behavioral Health and Primary Care Integration [10] and includes domains of work flow, clinical services, workspace, shared care and integration, case identification, and patient engagement. Domain scores and a total integration score are provided, along with median scores of other practices evaluated with the PIP.

\section{Results}

\section{EHR tracking}

The software program IBM SPSS for Macintosh version 23 was used to analyze data, including descriptive and inferential statistics from the initial dataset.

Demographics: Patient demographics and tracking outcomes were collected from the EHR during the initial 9-month pilot test of the FMBH program. The pilot included 188 initial new patient contacts and 80 patient followup visits with a BHC. The age of the patients ranged from 15 to 83 years, with a mean age of 44.54 years (standard deviation 15.86 years). Most patients were female (68.4\%).
The patients' reported race/ethnicity was 1.0\% Asian, 3.2\% American Indian/Alaska Native, 4.2\% Hispanic/Latino, $2.6 \%$ African American, and $75.5 \%$ white $(13.5 \%$ did not report their race/ethnicity).

Patient contact: In terms of follow-up frequencies, patient encounters ranged from one to eight visits, including the initial warm handoff encounter. The modal number of visits was 1 , with slightly under half of patients returning for at least one follow-up visit $(n=80)$. The amount of contact time per visit ranged from 5 to 90 minutes, and initial visits were slightly shorter on average than follow-up visits. Initial contacts were typically completed in 15-30 minutes versus 30 minutes for follow-up visits. See Table 1 for a further breakdown of patient contact.

Reasons for referral: Of the referrals received from primary care providers to the FMBH program, slightly under half of patients were referred primarily for mental health symptoms (e.g., anxiety, depression). Approximately one-third of patients were referred primarily for medical symptoms or behavioral medicine issues (e.g., headaches, diabetes), and the remainder of referrals included requests to address both mental and physical health issues. See Table 2 for a further breakdown of the reasons for referral.

Table 1. Patient contact and reason for referral

\begin{tabular}{|c|c|c|c|c|c|}
\hline & Number & Percentage & Range & Mean & SD \\
\hline \multicolumn{6}{|l|}{ Reason for referral } \\
\hline Mental health & 78 & 44 & & & \\
\hline Medical & 58 & 33.1 & & & \\
\hline Both & 39 & 22.3 & & & \\
\hline \multicolumn{6}{|l|}{ Patient contact } \\
\hline Number of visits & 188 & & $1-8$ & 2.43 & 1.77 \\
\hline \multicolumn{6}{|c|}{ Encounter time (minutes) } \\
\hline All visits & 188 & & $5-90$ & & \\
\hline Initial visits & 188 & & - & 34.12 & 16.36 \\
\hline Follow-up visits & 80 & & & 34.63 & 12.90 \\
\hline
\end{tabular}

The reason for referral indicates the primary issue for which the patient was referred to the Family Medicine-Behavioral Health program by the primary care provider. The number of visits indicates the number of unique patient encounters with the Family Medicine-Behavioral Health program during the pilot period. The encounter time indicates the face-to-face contact time with patients.

$\mathrm{SD}$, standard deviation. 
Table 2. Patient survey and clinical outcomes

\begin{tabular}{lrrrr}
\hline & Number & Mean & SD & Percentage \\
\hline PHQ-9 & & & & \\
$\quad$ Initial encounter & 21 & 12.76 & 6.99 & \\
$\quad$ Final encounter & 21 & $8.57^{*}$ & 7.41 & \\
Patient survey & & & & \\
$\quad$ Overall satisfaction & 7 & 4.6 & & 71.4 \\
First visit with BHC & 5 & & & 85.7 \\
PCP's idea to see BHC & 6 & & & 100 \\
Wait time less than & 7 & & & \\
20 minutes & & & & \\
\hline
\end{tabular}

Nine-item Patient Health Questionnaire (PHQ-9) scores collected at warm handoff versus final behavioral health consultant (BHC) encounter are reported. Patient survey overall satisfaction was measured on a five-point Likert scale. Patients reported whether or not the BHC encounter was the first visit with a BHC, whose idea the referral was, and wait time to see the BHC. See Appendix B for the full questionnaire.

*Statistically significant decrease at $P=0.011$.

PCP, primary care physician; SD, standard deviation.

\section{Initial patient outcomes: PHQ-9: Of the 21 initial patients} who completed the PHQ-9 at both the initial encounter and the final encounter, $85.7 \%$ screened positive for major depressive disorder on the PHQ-9 (a score of 5 or more in the previous 2 weeks). Of these patients, the mean total PHQ-9 scores represented a statistically significant decrease between assessments $\left(t_{20}=2.82, P=0.011\right)$. These PHQ-9 score changes, although limited, indicate that patients tended to report fewer mood symptoms after being seen in the FMBH program. However, it is important to note that we did not compare these findings with those for usual care, and thus these limited results must be interpreted with caution. See Table 2 for a further breakdown of PHQ-9 scores.

\section{Patient satisfaction}

Patient feedback was requested after the initial warm handoff encounter. Of patients who responded during the pilot phase, most indicated it was their physician's idea to see the BHC, most indicated that it was their first time seeing a behavioral health provider, and all of the initial patient responses indicated that their wait time to see a BHC was less than 20 minutes. The initial responses suggested overall patient satisfaction with the FMBH services received following a warm handoff (average total score of 4.6 on a five-point Likert scale). Although there has been a low initial response rate for patient satisfaction following warm handoffs and there is a potential for selection bias, these initial data suggest the potential for reaching patients who would not otherwise have been seen and for patients being satisfied with the behavioral health service. See Table 2 for a further breakdown of patient survey scores.

\section{Provider satisfaction and use}

During our initial pilot phase, primary care providers $(n=13)$ reported using FMBH services an average of one or two times per week and providers who have used these services indicated overall satisfaction with the FMBH program (average overall satisfaction score of 4.7 on a five-point Likert scale). Regression analysis results indicated a predictive relationship between primary care providers' satisfaction with the integrated behavioral health model and their use of these services, $F(1,12)=16.244, P=0.002$, adjusted $R^{2}=0.56$, suggesting that understanding which program components are conducive to provider satisfaction can play an important role in maximizing integrated service use (see Table 3).

\section{Practice integration}

The results of the PIP [9] suggest that the FMBH program is moderately integrated and scored closely to the median

Table 3. Summary of results of linear regression for provider satisfaction and use of Family Medicine-Behavioral Health program services

\begin{tabular}{lrrr}
\hline Predictor variable & B & $\begin{array}{r}\text { Service use } \\
\text { SE }\end{array}$ & $\beta$ \\
\hline Overall satisfaction & 0.142 & 0.035 & $0.772^{*}$
\end{tabular}

Overall satisfaction with the Family Medicine-Behavioral Health program indicates providers' response on a five-point Likert scale. Service use indicates the frequency of referral to the Family Medicine-Behavioral Health program on a five-point Likert scale. $* P=0.002$.

SE, standard error. 
of other practices. Notably lower than average development scores were obtained for case identification and patient engagement, and a notably higher than average development score was obtained for workspace, which indicated a fully developed level of integration within the FMBH program in terms of shared practice workspace.

\section{Discussion}

Despite limited resources, we were able to pilot a service program that has appeared to begin to meet the behavioral health service needs at the clinic. The initial findings are suggestive of provider use of and satisfaction with FMBH services, patient satisfaction with services, and clinical improvement. Practice integration findings indicate a moderate level of integration with the program so far, with shared clinic workspace being most noteworthy in regard to the level of integration. The physical proximity of the BHCs in the clinic (i.e., office space in the same wing as other providers; clinic space in the medical examination rooms) appears to be a key factor in the moderate success of the FMBH program thus far.

On the basis of our development, implementation, and initial outcomes of a novel integrated primary care-behavioral health program in our local community, it appears that the FMBH program has the potential to continue to serve an important role in addressing the behavioral health needs of the population. Interior Alaska is a rural and remote area that is largely underserved by behavioral health providers; there are limited resources to serve a relatively high need mental health population. As such, it has been a struggle to provide adequate care to behavioral health patients in this region because of the small population size and geographic remoteness. One of the benefits of the FMBH program we have designed is its ability to provide behavioral health services to those who would otherwise go underserved. For example, there are very few resources for Medicaid patients in our community, and behavioral health patients with Medicare or Medicaid are not always able to receive services, an issue faced by many rural and underserved communities [11]. One of the unforeseen benefits of piloting the FMBH program as a training model is that, because it is a training model, we have not billed for patient services and thus are able to capture a larger number of underserved patients than we anticipated.

\section{Future directions}

The initial outcomes currently presented are limited in scope and must be interpreted with caution; however, they are suggestive of the potential for promising longer-term outcomes. The next phase of our FMBH program development and implementation will include a comprehensive program evaluation. Our initial outcomes will help inform the next phase, which will include identifying and tracking specific, culturally relevant clinical health outcomes to be monitored throughout continued program implementation and evaluation (e.g., tobacco use, medication adherence, weight management, hemoglobin $\mathrm{A}_{1 \mathrm{c}}$ level). Once additional outcomes are identified, they will be added to ongoing assessment and tracking. See Table 1 for additional elements that will be included in the full FMBH program evaluation. We also plan to continue to evaluate the provider perspective in our next phase of program evaluation. Most of the current research for integrated models has focused on patient outcomes, which is understandable; however, the provider perspective is also important [12]. If we can better understand primary care providers' reasons for using FMBH services, we can better address barriers (i.e., education about the model, experience with the model) that may limit use as well as enhance potential providerspecific benefits of an integrated model. Finally, we hope to be able to demonstrate similar clinical and cost-effectiveness shown in other integrated healthcare systems [1] with our next phase of program evaluation. Included in our next steps is the evolution into a model that will fit into a sustainable billing system so as to capture the underserved population of patients with behavioral health service needs.

Our initial implementation data will also help provide support in the development of sustainable models from which integrated behavioral health program implementation can be guided throughout other rural regions in the United States. In addition to guiding sustainable models of integrated healthcare service delivery, the long-term program goals include implementing formalized provider training for behavioral health clinicians in the integrated healthcare model.

\section{Suggested applications}

Implementation of new service model designs can be variable depending on multiple factors, such as geographic location, need for services, the healthcare system, and the fee system. 
Our implementation design and initial program outcomes suggest that such models can work in small-scale settings with minimal overhead costs. In our case, it was especially helpful to have initial buy-in from the primary care providers, who helped advocate the program development to the clinic administration. We would encourage other small practices that are seeking to develop integrated primary care-behavioral health services, especially those in rural and remote settings, to consider an implementation design similar to ours.

\section{Conflict of interest}

The authors declare no conflict of interest.

\section{Funding}

This research received no specific grant from any funding agency in the public, commercial, or not-for-profit sectors.

\section{Author contributions}

Kendra Campbell contributed to project conceptualization, methods, investigation, supervision, project administration, data analysis, and writing. Angel Vasquez contributed to project methods, investigation, data analysis, and writing. Loren McKnight contributed to project methods, investigation, data analysis, and writing.

\section{References}

1. Blount A, Schoenbaum M, Kathol R, Rollman B, Thomas, M, Odonohue W. The economics of behavioral health services in medical settings: a summary of the evidence. Professional Prof Psychol Res Pr 2007;38:290-7.

2. Landis SE, Barrett M, Galvin SL. Effects of different models of integrated collaborative care in a family medicine residency program. Fam Syst Health 2013;31:264-73.
3. Talen M, Valeras AB. Integrated behavioral health in primary care: evaluating the evidence, identifying the essentials. New York: Springer; 2013. doi: 10.1007/978-1-4614-6889-9.

4. Woltmann E, Grogan-Kaylor A, Perron B, Georges H, Kilbourne AM, Bauer MS. Comparative effectiveness of collaborative chronic care models for mental health conditions across primary, specialty, and behavioral health care settings: systematic review and meta-analysis. Am J Psychiatry 2012;169:790-804.

5. World Health Organization. The world health report 2001. Mental health: new understanding, new hope. Geneva: World Health Organization; 2001.

6. Robinson P, Reiter J. Behavioral consultation and primary care: a guide to integrating services. New York: Springer; 2006.

7. Heath B, Wise Romero P, Reynolds K. A review and proposed standard framework for levels of integrated healthcare. Washing ton: SAMHSA-HRSA Center for Integrated Health Solutions; 2013.

8. Spitzer RL, Kroenke K, Williams JB. Validation and utility of a self-report version of PRIME-MD: the PHQ primary care study. J Am Med Assoc 1999;282:1737-44.

9. Kessler RS. Evaluating the process of mental health and primary care integration profile. Fam Med Community Health 2015;3:63-5.

10. Peek CJ, National Integration Academy Council. Lexicon for behavioral health and primary care integration: concepts and definitions developed by expert consensus. Rockville: Agency for Healthcare Research and Quality; 2013.

11. Mauksch LB, Tucker SM, Katon WJ, Russo J, Cameron J, Walker E, et al. Mental illness, functional impairment, and patient preferences for collaborative care in an uninsured, primary care population. J Fam Pract 2001;50:41-7.

12. Miller-Matero LR, Dykuis KE, Albujoq K, Martens K, Fuller BS, Robinson V, et al. Benefits of integrated behavioral health services: the physician perspective. Fam Syst Health 2016;34:51-5. 
Appendix A: Family Medicine-Behavioral Health program outcome tracking

\begin{tabular}{|c|c|c|}
\hline Clinic-specific information & Patient-specific information & Provider-specific information \\
\hline Use of BHC services by the clinic & Satisfaction with integrated health services** & Satisfaction with model ${ }^{*}$ \\
\hline Number of "warm handoff” referrals" & Demographic information* & Use of BHC services by provider ${ }^{*}$ \\
\hline Reasons for referral ${ }^{*}$ & Health issues/diagnoses ${ }^{* *}$ & \\
\hline Patient access to care & Follow-up disposition $^{* *}$ & \\
\hline Wait time to see $\mathrm{BHC}^{* *}$ & Type of intervention provided ${ }^{* *}$ & \\
\hline $\begin{array}{l}\text { Patient follow through on outside } \\
\text { mental health referrals }\end{array}$ & Health outcomes*,** & \\
\hline Degree of integration* & & \\
\hline
\end{tabular}

*Included in initial outcome data.

${ }^{* *}$ Will be included in full program evaluation.

BHC, behavioral health consultant.

\section{Appendix B: Family Medicine-Behavioral Health patient satisfaction survey}

Please circle the response that best reflects your experience with the family medicine-behavioral health consultants.

\begin{tabular}{|c|c|c|}
\hline $\begin{array}{l}\text { Whose idea was it to see } \\
\text { the behavioral health } \\
\text { consultant today? }\end{array}$ & $\begin{array}{l}\text { Was this your first time } \\
\text { seeing a behavioral } \\
\text { health provider? }\end{array}$ & $\begin{array}{l}\text { How long did you wait to see the } \\
\text { behavioral health consultant after } \\
\text { your primary care appointment? }\end{array}$ \\
\hline My idea & Yes & 0-20 minutes \\
\hline Nurse/physician & No & $21-40$ minutes \\
\hline Spouse/partner & & $41-60$ minutes \\
\hline \multirow[t]{2}{*}{ Other } & & $1-2$ hours \\
\hline & & 2-4 hours \\
\hline
\end{tabular}


Please circle the number that corresponds with your experience with the family medicine-behavioral health consultants.

1. Did your primary care provider and behavioral health consultant work well together?

$\begin{array}{ccccc}1 & 2 & 3 & 4 & 5 \\ \text { Disagree } & \text { Slightly disagree } & \text { Neutral } & \text { Slightly agree } & \text { Agree }\end{array}$

2. Did the behavioral health provider understand your problem?

$\begin{array}{ccccc}1 & 2 & 3 & 4 & 5 \\ \text { Disagree } & \text { Slightly disagree } & \text { Neutral } & \text { Slightly agree } & \text { Agree }\end{array}$

3. Are you confident in your ability to change the issue you saw the behavioral health provider for today?

$\begin{array}{ccccc}1 & 2 & 3 & 4 & 5 \\ \text { Disagree } & \text { Slightly disagree } & \text { Neutral } & \text { Slightly agree } & \text { Agree }\end{array}$

4. Were you satisfied with the advice given on ways to stay healthy or address your behavioral health needs?

$\begin{array}{ccccc}1 & 2 & 3 & 4 & 5 \\ \text { Disagree } & \text { Slightly disagree } & \text { Neutral } & \text { Slightly agree } & \text { Agree }\end{array}$

5. Did the behavioral health consultant explain things in a way you could understand?

$\begin{array}{ccccc}1 & 2 & 3 & 4 & 5 \\ \text { Disagree } & \text { Slightly disagree } & \text { Neutral } & \text { Slightly agree } & \text { Agree }\end{array}$

6. Was the behavioral health consultant willing to listen carefully to you?

$\begin{array}{ccccc}1 & 2 & 3 & 4 & 5 \\ \text { Disagree } & \text { Slightly disagree } & \text { Neutral } & \text { Slightly agree } & \text { Agree }\end{array}$




\section{Appendix C: Provider satisfaction and use survey}

1. With 1 being "not at all comfortable" and 5 being "very comfortable," how comfortable are you in addressing the mental health needs of your patients (without collaboration from a behavioral health provider)?

2. Please indicate your average use of Family MedicineBehavioral Health services (a warm handoff, electronic referral, or case consultation).

Not at all (1)

Monthly or less (2)

2-3 times per month (3)

1-2 times per week (4)

$\geq 3$ times per week (5)

3. What is your primary way of contacting the Family Medicine-Behavioral Health team?

Face-to-face (1)

Telephone task (2)

Paper referral (3)

Other (4)

4. How satisfied are you with the accessibility of the Family Medicine-Behavioral Health team?

Dissatisfied (1)

Somewhat dissatisfied (2)

Neutral (3)

Somewhat satisfied (4)

Satisfied (5)

5. How satisfied are you with our response time (i.e., how long did your patient wait to be seen by the Family Medicine-Behavioral Health team)?

Dissatisfied (1)

Somewhat dissatisfied (2)

Neutral (3)

Somewhat satisfied (4)

Satisfied (5)
6. How satisfied are you with the level of collaboration/ communication with the Family Medicine-Behavioral Health team?

Dissatisfied (1)

Somewhat dissatisfied (2)

Neutral (3)

Somewhat satisfied (4)

Satisfied (5)

7. How satisfied are you with observed patient outcomes following contact with the Family Medicine-Behavioral Health team?

Dissatisfied (1)

Somewhat dissatisfied (2)

Neutral (3)

Somewhat satisfied (4)

Satisfied (5)

8. How satisfied are you with the efficiency of referring your patients to the Family Medicine-Behavioral Health team?

Dissatisfied (1)

Somewhat dissatisfied (2)

Neutral (3)

Somewhat satisfied (4)

Satisfied (5)

9. How satisfied are you with the care provided for your patients by the Family Medicine-Behavioral Health team?

Dissatisfied (1)

Somewhat dissatisfied (2)

Neutral (3)

Somewhat satisfied (4)

Satisfied (5) 
10. How satisfied are you with the timeliness of communication concerning your patients with the Family Medicine-Behavioral Health team?

Dissatisfied (1)

Somewhat dissatisfied (2)

Neutral (3)

Somewhat satisfied (4)

Satisfied (5)

11. How satisfied are you with medical record documentation provided by the Family Medicine-Behavioral Health team?

Dissatisfied (1)

Somewhat dissatisfied (2)

Neutral (3)

Somewhat satisfied (4)

Satisfied (5)

12. How satisfied are you with the Family MedicineBehavioral Health services overall?

Dissatisfied (1)

Somewhat dissatisfied (2)

Neutral (3)

Somewhat satisfied (4)

Satisfied (5)

13. With 1 being "Not at all confident" and 5 being "Very confident," how confident are you that the Family Medicine-Behavioral Health team is equipped to care for your patients?

14. Please indicate on average how frequently you refer patients to a behavioral health provider other than the Family Medicine-Behavioral Health team.

Not at all (1)

Monthly or less (2)

2-3 times per month (3)

1-2 times per week (4)

$\geq 3$ times per week (5)
15. Below is a list of issues for which Family MedicineBehavioral Health services could be used. Please indicate which patient issues you have used the Family Medicine-Behavioral Health team for help with, through consultation, warm handoff, or referral (check all that apply).

\begin{tabular}{|c|c|c|}
\hline $\begin{array}{l}\text { Depression or mood } \\
\text { disorder }\end{array}$ & $\begin{array}{l}\text { Coping with } \\
\text { psychosocial } \\
\text { stress }\end{array}$ & $\begin{array}{l}\text { Substance abuse (alcohol } \\
\text { or drugs) }\end{array}$ \\
\hline Anxiety & $\begin{array}{l}\text { Coping with } \\
\text { medical illness } \\
\text { or diagnosis }\end{array}$ & Tobacco use \\
\hline PTSD & $\begin{array}{l}\text { Relational/ } \\
\text { family/parenting } \\
\text { problems }\end{array}$ & Weight issues \\
\hline $\begin{array}{l}\text { Acute distress/ } \\
\text { mental health crisis }\end{array}$ & Anger issues & Sleep problems \\
\hline Grief/bereavement & $\begin{array}{l}\text { Disruptive } \\
\text { behavior }\end{array}$ & $\begin{array}{l}\text { Behavioral support for } \\
\text { health issues or chronic }\end{array}$ \\
\hline Psychosis & Chronic pain & disease (e.g., diabetes) \\
\hline Suicidality & $\begin{array}{l}\text { Cognitive/ } \\
\text { memory issues }\end{array}$ & \\
\hline
\end{tabular}

